\title{
Is the Hemispheric Asymmetry of Monthly Sunspot Area an Irregular Process with Long-Term Memory?
}

\author{
Ratul Das ${ }^{1}$, Aparup Ghosh ${ }^{2}$, Bidya Binay Karak ${ }^{3,4 \star}$ \\ ${ }^{1}$ School of Physical Sciences, National Institute of Science Education and Research Bhubaneswar, Jatni, Khordha, Odisha, 752050, India \\ ${ }^{2}$ Department of Physical Sciences, Indian Institute of Science Education and Research Kolkata, Mohanpur, Nadia, West Bengal, 741246, India \\ ${ }^{3}$ Department of Physics, Indian Institute of Technology (Banaras Hindu University), Varanasi 221005, India \\ ${ }^{4}$ Max-Planck-Institut für Sonnensystemforschung, Justus-von-Liebig-Weg 3, D-37077 Goẗtingen, Germany
}

Accepted XXX. Received YYY; in original form ZZZ

\begin{abstract}
The hemispheric asymmetry of the sunspot cycle is a real feature of the Sun. However, its origin is still not well understood. Here we perform nonlinear time series analysis of the sunspot area (and number) asymmetry to explore its dynamics. By measuring the correlation dimension of the sunspot area asymmetry, we conclude that there is no strange attractor in the data. Further computing Higuchi's dimension, we conclude that the hemispheric asymmetry is largely governed by stochastic noise. However, the behaviour of Hurst exponent reveals that the time series is not completely determined by a memory-less stochastic noise, rather there is a long-term persistence, which can go beyond two solar cycles. Therefore, our study suggests that the hemispheric asymmetry of the sunspot cycle is predominantly originated due to some irregular process in the solar dynamo. The long-term persistence in the solar cycle asymmetry suggests that the solar magnetic field has some memory in the convection zone.
\end{abstract}

Key words: Sun: activity, sunspots, dynamo, magnetic fields — Time series analyses.

\section{INTRODUCTION}

The magnetic activity of Sun is not identical in two hemispheresthere is always an asymmetry. This hemispheric asymmetry, also called the north-south asymmetry, has been observed in the photospheric magnetic field (Mordvinov \& Kitchatinov 2004; McIntosh et al. 2013; Mordvinov \& Kitchatinov 2019) as well as in many proxies of the solar activity (Mandal et al. 2017; Goel \& Choudhuri 2009; Norton et al. 2014; Mordvinov et al. 2020). The hemispheric asymmetry is a real feature of the solar cycle and is not an artefact of inaccurate or noisy observations (Carbonell et al. 1993).

Bell (1961) found the evidence of hemispheric asymmetry in the number of major flares and later Bell (1962) found a long-term asymmetry in the sunspot area data during Cycles 8-18. Swinson et al. (1986) found a peak in the northern hemispheric solar activity about two years after sunspot minimum and a 22-years periodicity in the north-south asymmetry. Verma (1987) showed that the northern hemisphere is more active during Cycles 19 and 20. Li et al. (2009) used group sunspot and sunspot area data from 1996 to 2007 to show that the solar activity for cycle 23 is dominant in the southern hemisphere; also see Chowdhury et al. (2013) who extended this study to some part of cycle 24 . A well-known sunspot asymmetry was observed during the Maunder minimum. Most of the sunspots were registered in the southern hemisphere (Sokoloff \& Nesme-Ribes 1994).

It is believed that a hydromagnetic dynamo, operating in the solar

\footnotetext{
^ E-mail: karak.phy@iitbhu.ac.in
}

convection zone, is responsible for the generation and maintenance of the large-scale magnetic field and the cycle of solar activity (Parker 1955). In the current scenario of the solar dynamo (Karak et al. 2014; Charbonneau 2020; Hazra 2021), a toroidal component of the magnetic field is largely generated due to the shearing of the poloidal component by the differential rotation. This toroidal field gives back to the poloidal one due to the decay and dispersal of tilted bipolar magnetic regions (BMRs) - the so-called Babcock-Leighton process and possibly due to the helical convection-the so-called $\alpha$ effect. Meridional circulation and the small-scale convective flow play the role in transporting the magnetic field from the near-surface layer (the location of Babcock-Leighton process) to the deeper convection zone, where the shearing process is efficient, and thus largely regulates the cycle period.

The turbulent nature of the helical convective flow-the main drive of the dynamo-is expected to make the magnetic field unequal in two hemispheres. Thus, a hemispheric asymmetry in the solar magnetic field is unavoidable. Furthermore, the tilts of BMRs, which primarily determines the poloidal field, has a large scatter around Joy's law (Stenflo \& Kosovichev 2012; Wang et al. 2015; Arlt et al. 2016; Jha et al. 2020). Thus the tilt scatter makes the poloidal magnetic field irregular and asymmetric (Lemerle \& Charbonneau 2017; Karak \& Miesch 2017; Karak 2020). As the poloidal field is the seed for the toroidal field of the next solar cycle, the asymmetry in the polar field is propagated in the solar cycle (Choudhuri et al. 2007). Dynamo models have shown that when the turbulent diffusion is sufficiently strong, the coupling between two hemispheres tries to diminish the asymmetry introduced in the polar field 
and thus the asymmetry in the solar cycle may not persist for several cycles (Chatterjee \& Choudhuri 2006; Goel \& Choudhuri 2009; Karak 2010; Karak \& Miesch 2017). Dynamo models by including scatter in the BMR tilt (Lemerle \& Charbonneau 2017; Karak \& Miesch 2017, 2018) or $\alpha$ term in the poloidal source (Olemskoy \& Kitchatinov 2013; Karak et al. 2018; Hazra \& Nandy 2019) produce hemispheric asymmetry in the magnetic cycle, which in some parameter regimes, are in agreement with observations. Schüssler \& Cameron (2018) have shown that the random excitation of the quadrupole mode of dynamo by the stochastic fluctuations in the Babcock-Leighton process can lead to an asymmetry in the observed magnetic field; also see Nepomnyashchikh et al. (2019). Thus, all these previous results motivate us to explore whether the solar hemispheric asymmetry is governed by a low-dimensional chaotic process or stochastic process? Is there any long-term memory in the solar cycle asymmetry?

Nonlinear time series analysis is suitable to answer the above questions. While there exist many such studies for the solar cycle data (e.g., Ostriakov \& Usoskin 1990a; Carbonell et al. 1994; Jevtić et al. 2001; Letellier et al. 2006; Suyal et al. 2009), only a few such studies are performed in the solar cycle asymmetry. Carbonell et al. (1993) computed the correlation dimension of the asymmetry of daily sunspot area during 1874-1989 and did not find any evidence of lowdimensional chaos. By computing the Higuchi's fractal dimension (Higuchi 1988) and some nonlinear prediction method for the hemispheric asymmetry of sunspot number during 1947-1984, Watari (1996) concluded that the sunspot number asymmetry is highly irregular and not deterministic chaos.

In the present work, we shall utilize the maximum available sunspot area data (during 1874-2016) of hemispheric asymmetry and apply multiple nonlinear time series techniques to check the inherent nonlinear properties of the system. First, we shall compute the correlation dimension $\left(D_{2}\right)$ to extract whether the data has any strange attractor in the data and thus this analysis will reveal the existence of any low-dimensional chaos in the underlying system (Grassberger \& Procaccia 1983a). Next, we shall compute a fractal dimension using the method given in Higuchi (1988) which provides a stable estimate of the fractal dimension when the data is more irregular and non-stationary. Higuchi's dimension will give another independent support of whether the asymmetry data is from a stochastic process or low dimensional chaos. Finally, we shall compute the Hurst exponent (Mandelbrot \& Wallis 1969) to check whether the data has any persistent memory or not. The final conclusion will be presented in Section $\S 6$.

\section{OBSERVATIONAL DATA}

We use the monthly mean sunspot area data during May 1874 September 2016 obtained from the Royal Greenwich Observatory (RGO) ${ }^{1}$. The RGO data have been the only available record of sunspot area separately in two hemispheres for the longer duration and this is routinely used in many studies of the solar activity (Hathaway 2015). RGO provides the monthly value of the average (over observed day) of the daily sunspot area in the unit of millionths of a hemisphere and it is evenly spaced. We have also repeated our analyses with the newly available monthly mean hemispheric sunspot number data; $\S B$.

The easy way to measure the asymmetry is to take the difference

\footnotetext{
1 https://solarscience.msfc.nasa.gov/greenwch.shtml
}

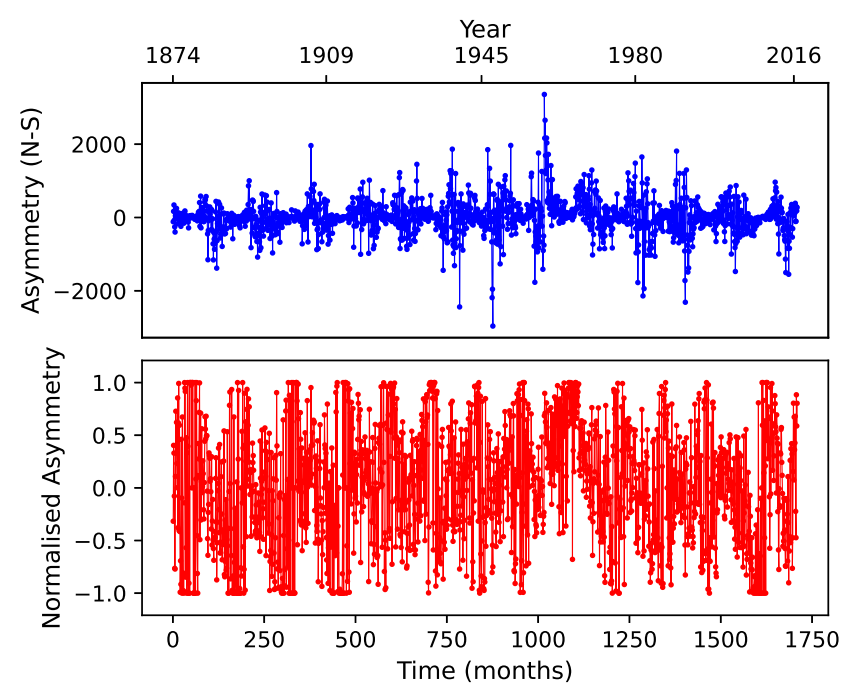

Figure 1. Time series of the hemispheric asymmetry of monthly average sunspot area (in unit of millionth of a solar hemisphere) as measured by $A S$ (top) and $A S_{\text {Norm }}$ (bottom).

in the values between two hemispheres (Ballester, J. L. et al. 2005; Chang 2007), i.e. asymmetry,

$A S=A_{N}-A_{S}$,

where $A_{N}$ and $A_{S}$ are the monthly values of the sunspot area in the northern and southern hemispheres, respectively. We note that during solar maxima, the difference becomes large in comparison to the value during minima and this causes a cyclic pattern in the asymmetry; see Figure 1 top panel. Furthermore, if a cycle is strong, then the asymmetry is large and vice versa. Therefore, in the literature (Carbonell et al. 1993; Oliver \& Ballester 1996; Duchlev 2001; Goel \& Choudhuri 2009; Chowdhury et al. 2013; Priyal et al. 2014), the asymmetry is also measured by normalizing its strength, namely. the normalized asymmetry,

$A S_{N o r m}=\frac{A_{N}-A_{S}}{A_{N}+A_{S}}$.

When both $A_{N}$ and $A_{S}$ become equal to zero, we set $A S_{N o r m}=0$. We realized that this happens for 15 data points (which is less than $1 \%$ of the total data). However, we discussed its effect in Section 4 by replacing these points with interpolated values.

We note that this $A S_{N \text { orm }}$ is a different time series than the $A S$; statistics of the data are different ( Table 1). Again this definition of asymmetry is not satisfactory because during the solar minima, when the sunspot area becomes very small and this leads to increase in $A S_{N \text { orm }}$; see Figure 1 bottom panel. It was examined in Yi (1992), that dividing the difference of the hemispherical sunspot areas by the total sunspot area results in the appearance of a peak in the power spectrum between 11 and 12 years.

Due to such facts from the literature, where both definitions have been used to measure the solar cycle asymmetry, it becomes necessary to perform analyses of the time series of asymmetry using both the methods that we have discussed above.

\section{METHODS}

To identify the nonlinear properties of the hemispheric asymmetry of sunspot area time series, we shall compute three quantities for $A S$ and 
Table 1. Some statistics of the data. Total number of data points used: 1709 . Symbols in second to third columns are as follows: $\langle S\rangle$, the average of the time series; $r m s$, the root mean-squared deviation, and $\langle\mathrm{PN}\rangle=\sqrt{\langle S\rangle}$.

\begin{tabular}{lrrc}
\hline \hline Data & $<S>$ & $r m s$ & $<\mathrm{PN}>/$ rms \\
\hline North & 426.79 & 488.2 & 0.042 \\
South & 409.43 & 470.1 & 0.043 \\
AS & 17.36 & 466.9 & 0.009 \\
AS & 0.01 & 0.6 & 0.225 \\
\hline
\end{tabular}

$A S_{\text {Norm }}$, namely, the Correlation dimension, Higuchi's dimension, and Hurst exponent. Below, we discuss briefly how to compute these quantities.

\subsection{Correlation dimension}

First, we shall apply a method of time series analysis to distinguish the random noise in the underlying system from the low dimensional chaos. This method is to measure a fractal dimension of the strange attractor in the system which is popularly known as the correlation dimension $\left(D_{2}\right)$. The method for obtaining $D_{2}$ has been given in Grassberger \& Procaccia (1983a,b), also see Takens (1981); Packard et al. (1980). This has also been used in many astrophysical applications (e.g., Schreiber 1999; Misra et al. 2006; Karak et al. 2010), including identifying chaotic dynamics of the solar cycle data (Ostriakov \& Usoskin 1990b; Carbonell et al. 1994; Jevtić et al. 2001). In this method, we construct a $M$ dimensional phase space using our time series $X(i)$ (where $i=1,2,3 \ldots . . N$ ); $X(i)$ is $A S$ or $A S_{\text {norm }}$ in our case. In this space, any vector has the following form:

$x_{i}=[X(i), X(i+\tau), \ldots \ldots \ldots, X(i+(M-1) \tau)]$.

The time delay, $\tau$ is chosen in such a way that each component becomes independent of each other. We find that at $\tau=3$ months, the auto-correlation of the data falls below $1 / e$ and thus we set this value for $\tau$ in our analysis (see Carbonell et al. 1994, for a detailed discussion on choosing $\tau$ ). We checked that our results do not change abruptly if we increase $\tau$.

Next, the correlation function $C_{M}(r)$ is given as

$C_{M}(r)=\frac{1}{N\left(N_{c}-1\right)} \sum_{i=1}^{N} \sum_{(j, j \neq i)}^{N_{c}} \Theta\left(r-\left|x_{i}-x_{j}\right|\right)$,

where $x_{i}$ is a reconstructed vector; Equation (3), $\Theta$ is Heaviside function $(\Theta(z)=1$ if $z \geq 0$ and 0 if $z<0), N$ the total number of points and $N_{c}$ is the number of centers. Essentially, $C_{M}(r)$ gives the number of points that are within a distance $r$ from the centre, averaged over all the centres. Then for small $r, D_{2}(M)$ is given by

$D_{2}(M)=\frac{\operatorname{dlog}\left(\mathrm{C}_{\mathrm{M}}(\mathrm{r})\right)}{\mathrm{d} \log (\mathrm{r})}$.

To compute $C_{M}(r)$, we divide the entire phase space into $M$ cubes of length $r$ around a point and count the average number of points. To avoid the edge effects due to the finite number of data points, we compute $C_{M}(r)$ in the range $r_{\text {min }}<r<r_{\text {max }}$. Here $r_{\text {min }}$ is chosen when $C_{M}(r)$ is just greater than one and $r_{\text {max }}$ is taken in such a way that all $M$ cubes remain within the embedding space. For a fixed value of $M, D_{2}$ is computed for different values of $r$ in the linear region of $\log \left(C_{M}(r)\right)$ versus $\log (r)$ plot using Equation (5). The average of all these values will give our final $D_{2}$ and the mean standard deviation over the average value gives the error on $D_{2}$. The whole calculation is repeated for different values of $M$.
The value of $D_{2}$ should increase initially with the increase of $M$. However, if the time series is obtained from a low dimensional chaotic system, then $D_{2}$ tends to saturate above a certain value of $M$. In contrast, for a stochastic system, $D_{2}$ keeps on increasing with $M$. That is the number of dimensions needed to describe the system in the phase space is infinitely large in a stochastic system. In that case, $D_{2} \approx M$, for all $M$. Thus, the variation of $D_{2}$ with $M$ is used to distinguish between the random noise vs low dimensional chaos.

\subsection{Higuchi's dimension}

Previously, many methods for finding stable estimations of the powerlaw spectral index have been discussed in the literature. Here, we follow the method given in Higuchi (1988) to calculate the fractal dimension $D$ of the asymmetry series. This method is helpful in providing a stable estimate of the fractal dimension of the asymmetry time series.

We recall that $X(i)$ is our time series $(i=1,2,3, \ldots, N ; N$ is the total number of observations taken at a regular interval) and thus,

$X(i): X(1), X(2), X(3), \ldots, X(N)$

From this, a new time series is constructed in the following manner:

$X_{\tau}^{m}: X(m), X(m+\tau), X(m+2 \tau), \ldots, X\left(m+\left[\frac{N-m}{\tau}\right] \tau\right)$

where $m=1,2, \ldots, \tau$, and [ ] denotes Gauss's notation. The length $L_{m}(\tau)$ of the curve associated to each $X_{\tau}^{m}$ is defined as:

$L_{m}(\tau)=\left\{\left(\sum_{i=1}^{\left[\frac{N-m}{\tau}\right]}|X(m+i \tau)-X(m+(i-1) \tau)|\right) \frac{N-1}{\left[\frac{N-m}{\tau}\right] \tau}\right\} \frac{1}{\tau}$,

The average value of the time series length $\langle L(\tau)\rangle$ for a given value of $\tau$ is defined as the average of $\tau$ sets of $L_{m}(\tau)$. If $\langle L(\tau)\rangle \propto \tau^{-D}$, for the range $\tau_{\min }<\tau<\tau_{\max }$ then the time series is a fractal and has a dimension $D$ for that range of $\tau$. We find $\langle L(\tau)\rangle$ for $\tau=2$ to $\tau=55$ for our analysis, for both $A S$ and for $A S_{N o r m}$.

\subsection{Hurst exponent}

Here, we attempt to find the Hurst exponent, $H$, which characterises the persistence of a times series to examine whether the non-periodic variations in the asymmetry time series are a result of a white noise process, an anti-correlated random process, or a correlated random process. We borrow the discussion regarding the Hurst exponent in the context of solar activity data from Ruzmaikin et al. (1994) and Suyal et al. (2009). We use the $R / S$ method as given in Mandelbrot \& Wallis (1969) to obtain the Hurst exponent.

We choose a temporal window $\tau$, where $\tau_{t}>\tau>N$, and $\tau_{t}$ is the Theiler window (Theiler 1986), to make subsets of time series $X(i)$ as follows:

$x_{i}(\tau) ; X\left(t_{0}\right), X\left(t_{0}+1\right), X\left(t_{0}+2\right), \ldots, X\left(t_{0}+\tau-1\right)$,

where $t_{0}=1,2, \ldots, N-\tau+1$. It is important to note that the choice of window used in finding the $R / S$ values for each given $\tau$ is rather important, since non-overlapping windows produce $R / S$ values from comparatively small sample sizes. Lesser number of windows could possibly provide inaccurate values for $H$. Now we denote the average of these subsets as:

$\bar{x}\left(t_{0}, \tau\right)=\frac{1}{\tau} \sum_{i=t_{0}}^{t_{0}+\tau-1} x_{i}(\tau)$. 


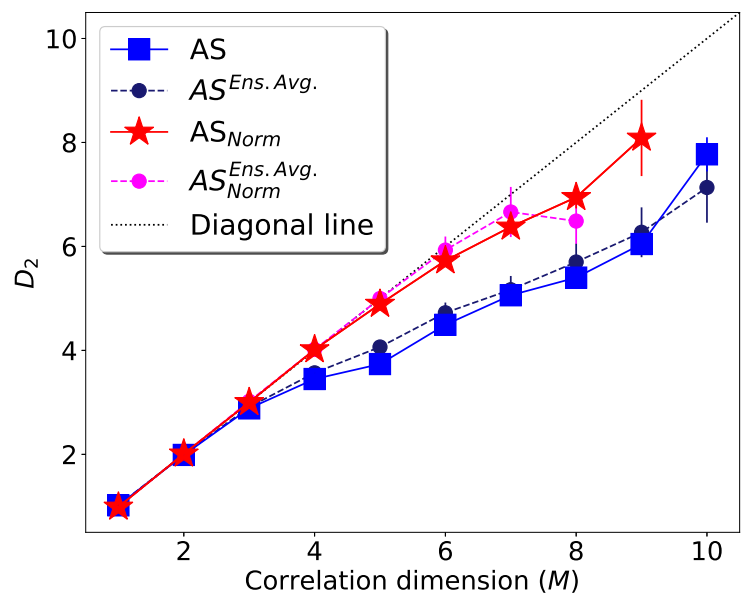

Figure 2. Variation of correlation dimension $\left(D_{2}\right)$ with the embedding dimension $(M)$ for $A S$ (square points) and $A S_{\text {Norm }}$ (asterisks). The dotted line along the diagonal of the figure indicates the $D 2$ variation as expected from an ideal stochastic process. $A S^{E n s . A v g}$. and $A S_{N o r m}^{\text {Ens.Avg. (round points) }}$ shows the variation averaged over an ensemble of $1000 \mathrm{AS} \& A S_{N \text { orm }}$ time series, respectively (see Section 5).

Let, $S\left(t_{0}, \tau\right)$ be the standard deviation of $x_{i}(\tau)$ for the window $\tau$ as follows:

$S\left(t_{0}, \tau\right)=\sqrt{\frac{1}{\tau-1} \sum_{i=t_{0}}^{t_{0}+\tau-1}\left[x_{\tau}^{t_{0}}(i)-\bar{x}\left(t_{0}, \tau\right)\right]^{2}}$.

Now, we define a set of new variables $y_{i}\left(t_{0}, \tau\right)$, which is the set of cumulative deviations from the mean of $x_{i}(\tau)$

$y_{i}\left(t_{0}, \tau\right)=\sum_{k=t_{0}}^{t_{0}+i-1}\left[x_{\tau}^{t_{0}}(k)-\bar{x}\left(t_{0}, \tau\right)\right]$,

and hence, the range $R$ of $y_{i}\left(t_{0}, \tau\right)$ is obtained as:

$R\left(t_{0}, \tau\right)=\max _{1 \leq i \leq \tau} y_{i}\left(t_{0}, \tau\right)-\min _{1 \leq i \leq \tau} y_{i}\left(t_{0}, \tau\right)$.

This allows us to define the rescaled range measure $R / S$ as:

$(R / S)\left(t_{0}, \tau\right)=\frac{R\left(t_{0}, \tau\right)}{S\left(t_{0}, \tau\right)}$.

Calculating the $R / S$ values for each temporal window by moving from $t_{0}=1$ to $t_{0}=N-\tau+1$ for window size $\tau$, the rescaled range for $\tau$ is then given as the average of these values

$(R / S)=\frac{1}{N-\tau+1} \sum_{t_{0}}(R / S)\left(t_{0}, \tau\right)$.

It was observed that the rescaled range for a time window is proportional to $\tau^{H}$

$(R / S)_{\tau}=k \tau^{H}$

where $k$ is the proportionality constant, and $H$ is the Hurst exponent. To obtain the value of the Hurst exponent, $R / S$ values are plotted for $\tau=11$ to $\tau=1709$ for the Hurst Exponent analysis, for both $A S$ and $A S_{\text {Norm }}$.

A white noise process or a random walk process is defined by a Hurst exponent of $H=0.5$. When the time series has $H>0.5$, it is said to be persistent. Persistence is defined as the tendency for the

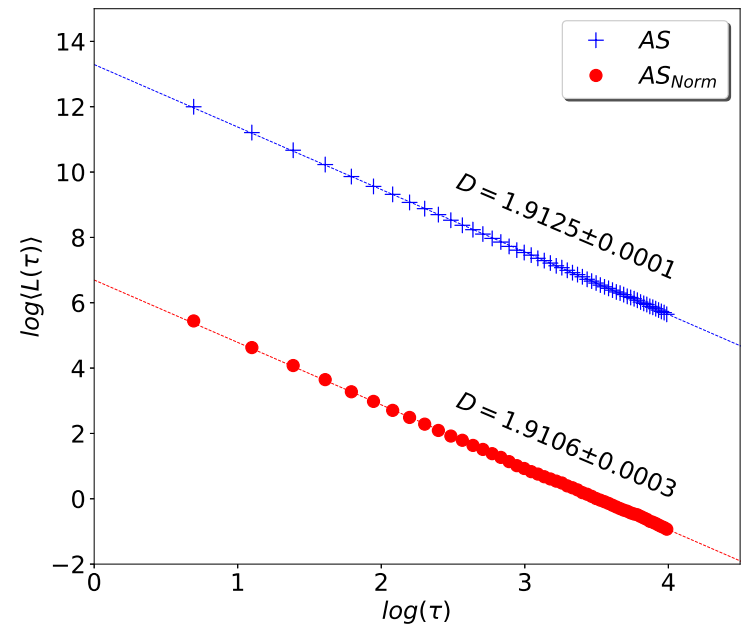

Figure 3. Variation of the length $(\langle L(\tau)\rangle)$ with the time interval $(\tau)$ for $A S$ and $A S_{N o r m}$.
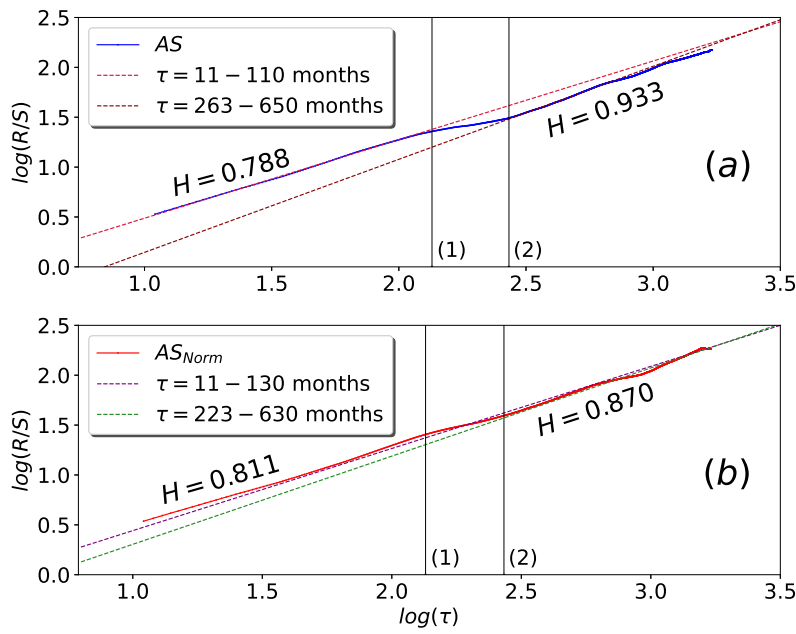

Figure 4. Variation of $R / S$ with time interval ( $\tau$ ) for $A S$ (top) and $A S_{N \text { orm }}$ (bottom). $\tau=135$ months and $\tau=271$ months are indicated as (1) and (2).

process to have a memory of the previous step. That is, if there was an increase in the value of the time series, the following step would be more likely to have an increase as well. In such a case, the time series would cover more "distance" than a random walk would. Whereas, if $0<H<0.5$, then the time series is said to be anti-persistent. That is, an increase in the value of the time series is more likely to be followed by a decrease, and vice-versa. And in opposition to a persistent case, the time series would cover less "distance" than a random walk.

There are other methods to determine the Hurst exponent, and the value that is obtained is sensitive to the method used (Weron 2002). In order to provide a confidence in the estimate of the Hurst exponent, and to ensure that the result is not method dependent, we shall compute Hurst exponent in two more methods, namely, Detrended Fluctuation Analysis and Periodogram Regression. As these methods are well described in the literature, we shall describe them briefly in Appendix A. 

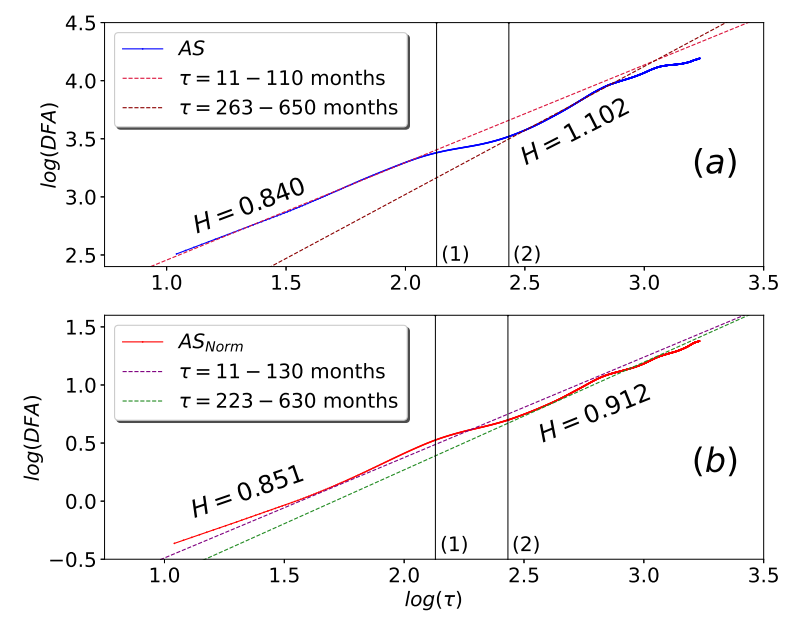

Figure 5. Variation of $D F A$ with time interval ( $\tau$ ) for $A S$ (top) and $A S_{N \text { orm }}$ (bottom). $\tau=135$ and $\tau=271$ months are indicated as (1) and (2).

\section{RESULTS}

The variation of $D_{2}$ as function of $M$ for the sunspot area asymmetry is shown in Figure 2. We find that the asymmetry and normalized asymmetry do not show the same behaviour. Nevertheless, in both cases, $D_{2}$ increases with an increase of $M$. The lack of saturation in $D_{2}$ implies that the sunspot area asymmetry is not governed by lowdimensional chaos, rather it might be driven by a high-dimensional or stochastic process. This conclusion is in general agreement with Carbonell et al. (1993) who also did not find the evidence of lowdimensional chaos in the asymmetry of sunspot area data during 1874-1989.

To confirm that the solar cycle asymmetry is really governed by stochastic or high-dimensional chaos, we observe the value of Higuchi's dimension $(D)$. As seen from Figure 3, for $A S$, $D=1.9125 \pm 0.0001$, and for $A S_{N o r m}, D=1.9106 \pm 0.0003$. We know that when the value of $D$ for a curve is close to 2 , the curve behaves nearly like a surface, i.e., the curve is close to a space-filling curve. Hence, the self-similar nature for time series, i.e., the hallmark of low-dimensional chaos is absent. Therefore, we conclude that the process that generates the hemispheric asymmetry of sunspot area is very likely to be the result of an irregular or stochastic process.

Finally, we explore the memory of these irregular asymmetry data by computing the Hurst exponent $(H)$. In Figure 4, we show the $\log$-log plots for $R / S$ against $\tau$. The slope of this curve gives the $\mathrm{H}$ value. We, however, see two distinguishable linear scaling regimes, and hence one value of $\mathrm{H}$ for all $\tau$ is not adequately representing the data. The previous study for sunspot number cycle also indicted two distinct regimes (Suyal et al. 2009).

For $A S$, in the range: $\tau=11-110$ months, we find $H=0.79$, while for $\tau=263-650$ months, we obtain $H=0.94$ (Table 2). In between these two regimes, there is a small region during 135-271 months (marked by vertical lines in (Figure 4a)) with a weaker slope, which is possibly linked to a period of lower persistence in the trend. For $A S_{N \text { orm }}$ (Figure $4 \mathrm{~b}$ ), in the range $\tau=11-130$ months, we find $H=0.81$, while in the range: $\tau=233-630$ months, we get $H=0.87$. In this case, the change in the slope happens very slowly.

In the Detrended Fluctuation Analysis (DFA), we get similar results but the values of $H$ are slightly larger (Figure 5). For $A S$, in the range: $\tau=1-110$ months, we find $H=0.84$, while for $\tau=263-650$ months, we obtain $H=1.10$. Now, as discussed in Bryce \& Sprague
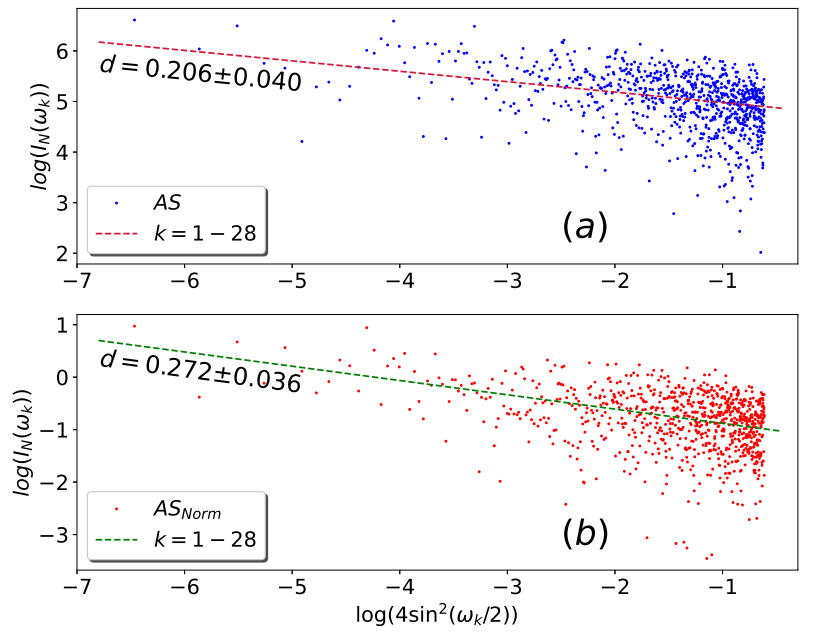

Figure 6. Periodogram Geweke-Porter-Hudak (GPH) Method for AS (top) and $A S_{\text {Norm }}$ (bottom).

(2012); Ceballos \& Largo (2017), we can point out that a value of $H$ above 1 is not impossible. It is a consequence of non-stationarity or a trend not being fully removed from the data. The reliability of DFA as a valid method has been questioned on similar grounds before. But since our R/S analysis still backs up the general result that the latter regime has a higher slope, we can safely negate the effect that this inconsistency may cause. (Table 2).

For $A S_{N \text { orm }}$, in the range: $\tau=11-130$ months, we find $H=0.85$, while in the range: $\tau=223-630$ months, we get $H=0.91$.

Using the Method of Periodogram Regression (Figure 6), for $A S$, we obtain $H(=0.5+0.21)=0.71$, while for $A S_{N \text { orm }}$, we obtain $H(=$ $0.5+0.27)=0.77$. Unlike the case in $R / S$ Method and Detrended Fluctuation Analysis, the periodogram (Figure 6) does not show two distinct linear scaling regimes. However, the Hurst exponent being larger than 0.5 , we can safely conclude that $A S$ and $A S_{N \text { orm }}$ time series are persistent in nature and the degree of persistence increases with the time-scale. Larger value of $H$ for $\tau \gtrsim 22$ years $(=264$ months), suggest that the memory of solar cycle asymmetry persists at least for two cycles.

We have obtained $H$ and $D$ graphically, using the Least Squares method for all of our plots; see e.g., Figure 3. We have also applied Bayesian linear regression to all our power law fits (Wheatland 2004) using the justification towards a Bayesian method of fitting as opposed to a frequentist method, as explained in D'Huys et al. (2016). We use the Python module, PyMC3 (Salvatier et al. 2016) for this. We find that the differences in results lie in the range of $10^{-4}-$ $10^{-3}$, except in the case of of the periodogram method. In that case, for $A S$ and $A S_{N \text { orm }}$ we obtain the value of the Hurst exponents as $0.707 \pm 0.179$ and $0.761 \pm 0.132$, respectively using Bayesian linear regression, while these values obtained from previous Least Squares method are 0.706 and 0.772 , respectively.

We recall that while computing $A S_{\text {Norm }}$ using Equation (2), we took $A S_{\text {Norm }}=0$ when both $A_{N}$ and $A_{S}$ are zero. Instead of this, if we replace these points by interpolating the neighbouring points, then this so-called zero-replacement strategy affects our computed results only marginally. (For example, from this zero-replacement $A S_{\text {Norm }}$ data, the computed value of $D$ is 1.9077 and the values of $H$ are $0.8110 \& 0.8693$ (R/S method), $0.8469 \& 0.9074$ (DFA), and 0.7718 (PR). Compare these values with the corresponding values in Table 2.) 
Table 2. Summary of results obtained for Higuchi's dimension and Hurst exponents. In the forth and sixth columns, the values are the means and the standard deviations (errors) of the results obtained from an ensemble of $1000 \mathrm{AS} \& \mathrm{AS}_{\text {Norm }}$ time series, respectively (see text).

\begin{tabular}{llcccc}
\hline \hline \multirow{2}{*}{ Method } & \multirow{2}{*}{$\begin{array}{l}\text { Window } \\
(\text { months })\end{array}$} & AS & Ensemble of AS & $\mathrm{AS}_{\text {Norm }}$ & Ensemble of AS $_{\text {Norm }}$ \\
\cline { 3 - 6 } & $2-55$ & 1.912 & $1.915 \pm 0.002$ & 1.911 & $1.916 \pm 0.002$ \\
\hline Higuchi & $11-110$ & 0.788 & $0.783 \pm 0.008$ & 0.811 & $0.813 \pm 0.007$ \\
Hurst (R/S) & $263-650$ & 0.933 & $0.929 \pm 0.019$ & 0.871 & $0.879 \pm 0.017$ \\
\hline Hurst (DFA) & $11-110$ & 0.840 & $0.834 \pm 0.010$ & 0.851 & $0.851 \pm 0.008$ \\
& $263-650$ & 1.102 & $1.096 \pm 0.022$ & 0.912 & $0.920 \pm 0.018$ \\
\hline Hurst (PR) & $\mathrm{K}=28$ & 0.706 & $0.704 \pm 0.039$ & 0.772 & $0.775 \pm 0.028$ \\
\hline
\end{tabular}

\section{ERROR ESTIMATES}

In our study, we used the monthly averaged hemispheric sunspot area as recorded in RGO. Unfortunately, in these data, no error information is given. Therefore we cannot make a direct estimate of error in our computed results. However, using the daily sunspot area data ${ }^{1}$, we can make some estimate of the errors in the following way using a Bootstrapping technique (Efron \& Tibshirani 1993). Let us consider the daily sunspot area data of one month for both the northern and southern hemispheres. We produce 100 resampled datasets with the same size as the number of days for this month by randomly selecting daily pairs of values for north and south, and then computing the corresponding $A S$ and $A S_{N \text { orm }}$ for the resamples. We compute the mean for all of these resamples. And then, we compute the mean $(\mu)$ and the standard deviation $(\sigma)$ of the means of all the resampled datasets for a month. It can be easily seen that this mean is not necessarily the same as what we have used in our earlier analyses. With these $\mu$ and $\sigma$, we produce an ensemble of 1000 data points (deviate) from a Gaussian distribution and repeat this for all the months to get 1000 time series. Finally, perform our all the nonlinear time series analyses with this ensemble.

Black and magenta filled circles connecting dashed lines in Figure 2 show the average $D_{2}$ behaviour of the ensemble of $1000 \mathrm{AS}$ and $A S_{\text {Norm }}$ time series. The error bar represents the $\sigma$ of the computed $D_{2}$ of 1000 time series. In Table 2, forth and sixth columns show the mean and error (standard deviation) of $D$ and $H$ from the ensembles.

We clearly see that the mean values of the computed quantities $\left(D_{2}\right.$, $D$, and $H$ ) of the ensemble of $1000 A S \& A S_{N o r m}$ time series are not too far from the ones computed from the original monthly mean time series. The values of $\sigma$ of the ensemble are also reasonably low, with the quantities being less than 1 standard deviation away from the computed values in most cases.

\section{DISCUSSION AND CONCLUSION}

We have explored some nonlinear properties of the underlying process behind the solar cycle asymmetry using nonlinear time series analysis. For this, we have used the hemispheric monthly sunspot area and number time series, which are the best proxies of the Sun's large-scale magnetic flux available for a longer duration.

Following the literature, solar cycle asymmetry has been measured in two ways, namely, the hemispheric difference $A S$ and the normalized hemispheric asymmetry $A S_{N \text { orm }}$. We have used three methods of time series analyses to characterise the data.

From the analysis of the correlation dimension $D_{2}$, we find that the value of $D_{2}$ does not saturate for higher values of $M$. This indicates that there is no underlying presence of a low-dimensional chaotic attractor that could govern the asymmetry of sunspot area data, in agreement with the conclusion obtained in Carbonell et al. (1993). In other words, we can expect that the asymmetry is likely to be produced by irregular process.

In our fractal analysis, we see that the value obtained for the Higuchi's fractal dimension $(D)$ is close to 2 , which implies that a stochastic process or possibly a high-dimensional chaotic process is the cause of the asymmetry.

In all three methods of computation of Hurst exponent, we find the value of Hurst exponent $H$ is above 0.7 for $A S$ data and a little larger for $A S_{\text {Norm}}$. We find multiple values of $H$ for the same time series. Its value decreases slightly after about 11 years (one cycle period) and then increases for windows larger than about 22 years. This change in the value of $\mathrm{H}$ and thus the persistence is more prominent in $A S$. In general, a memory can be observed for the asymmetry time series and it is larger in long-time scale (beyond 22 years; two cycles). From our analysis, we conclude that the monthly hemispheric asymmetry of sunspot area is dictated by a stochastic process with some amount of long-memory.

The results from hemispheric sunspot number, which is recently made available by Veronig et al. (2021) during 1874-2020, also shows similar behaviour (during the period May 1874 - September 2016) as that found in the hemispheric area data; $\S \mathrm{B}$.

Stochastic nature of hemispheric asymmetry supports the previous theoretical studies (e.g., Goel \& Choudhuri 2009; Olemskoy \& Kitchatinov 2013; Karak \& Miesch 2017; Schüssler \& Cameron 2018; Hazra \& Nandy 2019; Nepomnyashchikh et al. 2019) which explains the solar cycle asymmetry to be caused by the irregularity involved in the helical convective flow, and in particular the randomness involved in the Babcock-Leighton process (e.g., in the form of tilt of BMRs, emergence rate, meridional flow). Further, the presence of some long-term memory in asymmetry time series supports the existence of a finite memory of the sun's magnetic field, which is possibly determined by the turbulent diffusion and pumping (Chatterjee \& Choudhuri 2006; Karak \& Nandy 2012; Karak \& Miesch 2017; Kitchatinov \& Khlystova 2021).

\section{ACKNOWLEDGEMENT}

B.B.K. thanks Banibrata Mukhopadhyay, Jayanta Dutta and Vinita Suyal for many discussion on time series analysis and help in writing codes during his $\mathrm{PhD}$ time. He also thanks Bibhuti Kumar Jha and Prasun Dutta for the discussion on error analyses. Authors thank the anonymous referee who provided us valuable feedback on the ear- 
lier versions of this paper. B.B.K. acknowledges the funding from Department of Science and Technology (SERB/DST), India through the Ramanujan Fellowship (project no SB/S2/RJN-017/2018) and the support provided by the Alexander von Humboldt Foundation during a part of this project. A.G. acknowledges Kishore Vaigyanik Protsahan Yojana (KVPY) for scholarship. R.D. acknowledges the DAE Incentive Scheme for Holistic Science Education and Augmentation (DISHA) for scholarship.

\section{DATA AVAILABILITY}

Sunspot area data used in the present study is obtained from the Royal Greenwich Observatory; http://solarscience.msfc.nasa.gov/greenwch.shtml. Data of our analyses presented in the article will be shared upon reasonable request to the corresponding author.

\section{REFERENCES}

Arlt R., Senthamizh Pavai V., Schmiel C., Spada F., 2016, A\&A, 595, A104 Ballester, J. L. Oliver, R. Carbonell, M. 2005, A\&A, 431, L5 Bell B., 1961, Smithsonian Contributions to Astrophysics, 5, 69 Bell B., 1962, Smithsonian Contributions to Astrophysics, 5, 187 Bryce R. M., Sprague K. B., 2012, Scientific Reports, 2, 315 Carbonell M., Oliver R., Ballester J. L., 1993, A\&A, 274, 497 Carbonell M., Oliver R., Ballester J. L., 1994, A\&A, 290, 983 Ceballos R. F., Largo F. F., 2017, Imperial Journal of Interdisciplinary Research 2017), 3, 424

Chang H.-Y., 2007, Journal of Astronomy and Space Sciences, 24, 261-268 Charbonneau P., 2020, Living Reviews in Solar Physics, 17, 4

Chatterjee P., Choudhuri A. R., 2006, Sol. Phys., 239, 29

Choudhuri A. R., Chatterjee P., Jiang J., 2007, Physical Review Letters, 98, 131103

Chowdhury P., Choudhary D. P., Gosain S., 2013, ApJ, 768, 188

Duchlev P. I., 2001, Solar Physics, 199, 211-215

D’Huys E., Berghmans D., Seaton D. B., Poedts S., 2016, Solar Physics, 291, $1561-1576$

Efron B., Tibshirani R., 1993, An Introduction to the Bootstrap. Springer Science+Business Media, B. V.

Geweke J., Porter-Hudak S., 1983, J. Time Ser. Anal., 4, 221

Goel A., Choudhuri A. R., 2009, Research in Astronomy and Astrophysics, 9,115

Grassberger P., Procaccia I., 1983a, Physica D Nonlinear Phenomena, 9, 189

Grassberger P., Procaccia I., 1983b, Phys. Rev. Lett., 50, 346

Hathaway D. H., 2015, Living Reviews in Solar Physics, 12, 4

Hazra G., 2021, Journal of Astrophysics and Astronomy, 42, 22

Hazra S., Nandy D., 2019, MNRAS, 489, 4329

Higuchi T., 1988, Physica D Nonlinear Phenomena, 31, 277

Jevtić N., Schweitzer J. S., Cellucci C. J., 2001, A\&A, 379, 611

Jha B. K., Karak B. B., Mandal S., Banerjee D., 2020, ApJ, 889, L19

Karak B. B., 2010, ApJ, 724, 1021

Karak B. B., 2020, arXiv e-prints, p. arXiv:2009.06969

Karak B. B., Miesch M., 2017, ApJ, 847, 69

Karak B. B., Miesch M., 2018, ApJ, 860, L26

Karak B. B., Nandy D., 2012, ApJ, 761, L13

Karak B. B., Dutta J., Mukhopadhyay B., 2010, ApJ, 708, 862

Karak B. B., Jiang J., Miesch M. S., Charbonneau P., Choudhuri A. R., 2014, Space Sci. Rev., 186, 561

Karak B. B., Mandal S., Banerjee D., 2018, ApJ, 866, 17

Kitchatinov L. L., Khlystova A. A., 2021, ApJ

Lemerle A., Charbonneau P., 2017, ApJ, 834, 133

Letellier C., Aguirre L. A., Maquet J., Gilmore R., 2006, A\&A, 449, 379

Li K. J., Chen H. D., Zhan L. S., Li Q. X., Gao P. X., Mu J., Shi X. J., Zhu W. W., 2009, Journal of Geophysical Research (Space Physics), $114, \mathrm{~A} 04101$
Mandal S., Karak B. B., Banerjee D., 2017, ApJ, 851, 70

Mandelbrot B. B., Wallis J. R., 1969, Water Resources Research, 5, 321

McIntosh S. W., et al., 2013, ApJ, 765, 146

Misra R., Harikrishnan K. P., Ambika G., Kembhavi A. K., 2006, The Astrophysical Journal, 643, 1114

Mordvinov A. V., Kitchatinov L. L., 2004, Astronomy Reports, 48, 254

Mordvinov A. V., Kitchatinov L. L., 2019, Sol. Phys., 294, 21

Mordvinov A. V., Karak B. B., Banerjee D., Chatterjee S., Golubeva E. M., Khlystova A. I., 2020, arXiv e-prints, p. arXiv:2009.11174

Nepomnyashchikh A., Mandal S., Banerjee D., Kitchatinov L., 2019, A\&A, 625, A37

Norton A. A., Charbonneau P., Passos D., 2014, Space Sci. Rev., 186, 251

Olemskoy S. V., Kitchatinov L. L., 2013, ApJ, 777, 71

Oliver R., Ballester J. L., 1996, Solar Physics, 169, 215-224

Ostriakov V. M., Usoskin I. G., 1990a, Sol. Phys., 127, 405

Ostriakov V. M., Usoskin I. G., 1990b, Sol. Phys., 127, 405

Packard N. H., Crutchfield J. P., Farmer J. D., Shaw R. S., 1980, Phys. Rev. Lett., 45, 712

Parker E. N., 1955, ApJ, 122, 293

Peng C. K., Buldyrev S. V., Havlin S., Simons M., Stanley H. E., Goldberger A. L., 1994, Phys. Rev. E, 49, 1685

Priyal M., Banerjee D., Karak B. B., Muñoz-Jaramillo A., Ravindra B., Choudhuri A. R., Singh J., 2014, ApJ, 793, L4

Ruzmaikin A., Feynman J., Robinson P., 1994, Sol. Phys., 149, 395

Salvatier J., Wiecki T. V., Fonnesbeck C., 2016, PeerJ Computer Science, 2, e55

Schreiber T., 1999, Phys. Rep., 308, 1

Schüssler M., Cameron R. H., 2018, A\&A, 618, A89

Sokoloff D., Nesme-Ribes E., 1994, A\&A, 288, 293

Stenflo J. O., Kosovichev A. G., 2012, ApJ, 745, 129

Suyal V., Prasad A., Singh H. P., 2009, Sol. Phys., 260, 441

Swinson D. B., Koyama H., Saito T., 1986, Sol. Phys., 106, 35

Takens F., 1981, Detecting strange attractors in turbulence. p. 366, doi:10.1007/BFb0091924

Theiler J., 1986, Phys. Rev. A, 34, 2427

Verma V. K., 1987, Sol. Phys., 114, 185

Veronig A. M., Jain S., Podladchikova T., Pötzi W., Clette F., 2021, A\&A, 652, A56

Wang Y.-M., Colaninno R. C., Baranyi T., Li J., 2015, ApJ, 798, 50

Watari S., 1996, Sol. Phys., 163, 259

Weron R., 2002, Physica A Statistical Mechanics and its Applications, 312,285

Wheatland M. S., 2004, ApJ, 609, 1134

Yi W., 1992, Journal of the Royal Astronomical Society of Canada, 86, 89-98

\section{APPENDIX A: HURST EXPONENT USING DIFFERENT METHODS}

In this section, we will determine the Hurst exponent by two other methods, namely, Detrended Fluctuation Analysis and Periodogram Regression.

The method of Detrended Fluctuation Analysis proposed by Peng et al. (1994) and also discussed in Weron (2002) can be summarized as follows. We choose a temporal window $\tau$, where $\tau_{0} \leq \tau \leq N$. Here, $\tau_{0}$ plays a similar role as the Theiler window plays in the $R / S$ method. For this $\tau$, we can divide the time series $X(t)$ into $d(=N-\tau+1)$ subseries

$x_{i}(\tau): X(i), X(i+1), \ldots, X(i+\tau-1)$

for $i=1,2,3, \ldots, d$. Note that each subseries $x_{i}(\tau)$ is of length $\tau$. Now, for each subseries $x_{i}(\tau)$, we create a cumulative time series

$Y_{i}(\tau): X(i), \sum_{j=0}^{1} X(i+j), \ldots, \sum_{j=0}^{\tau-1} X(i+j)$. 
Now, we fit a least-squares line $\bar{Y}_{i}(\tau, x)=a_{i} x+b_{i}$ where, $x=$ $1,2, \ldots, \tau$, to $\left\{Y_{i}(\tau)\right\}$. Let, $S_{i}(\tau)$ be the root mean square fluctuation (i.e. standard deviation) of the Integrated and Detrended time series, given by

$S_{i}(\tau)=\sqrt{\frac{1}{\tau} \sum_{x=1}^{\tau}\left(Y_{i, x}(\tau)-a_{i} x-b_{i}\right)^{2}}$

where, $Y_{i, x}(\tau)$ represents the $\mathrm{x}$-th element of the cumulative time series $Y_{i}(\tau)$. Finally, we calculate the mean value of the root mean square fluctuation for all subseries of length $\tau,(D F A)_{\tau}$, given by

$(D F A)_{\tau}=\frac{1}{d} \sum_{i=1}^{d} S_{i}(\tau)$

Similar to the case of the $R / S$ analysis, a linear relationship on a double logarithmic paper of $(D F A)_{\tau}$ against $\tau$ indicates the presence of a power-law scaling

$(D F A)_{\tau}=k \tau^{H}$

where, $k$ is the proportionality constant and $H$ is the Hurst exponent. To obtain the values of the Hurst exponent, $(D F A)$ values are plotted for $\tau=11-1709$ months for both $A S$ and $A S_{N o r m}$.

Now, we will discuss the method of Periodogram Regression proposed by Geweke \& Porter-Hudak (1983). It is calcualted from the slope of the spectral density function of a fractionally integrated series at low frequencies.

For the time series, we start out by calculating the periodogram, which is a sample analogue of the spectral density. Given the time series $X(i)$ of length $N$, the periodogram is obtained as

$I_{N}\left(\omega_{k}\right)=\frac{1}{N}\left|\sum_{t=1}^{N} X(t) e^{-i 2 \pi(t-1) \omega_{k}}\right|^{2}$

where, $\omega_{k}=k / N, k=1,2, \ldots,[N / 2]$ and $[X]$ denotes the greatest integer less than or equal to $X$ Note that, $I_{L}$ is the squared absolute value of the Fourier transform. The next step is to run a linear regression

$\log \left\{I_{N}\left(\omega_{k}\right)\right\}=a-\hat{d} \log \left\{4 \sin ^{2}\left(\omega_{k} / 2\right)\right\}+\epsilon_{k}$

at low Fourier frequencies $\omega_{k}, k=1,2, \ldots, K \leq[L / 2] . \omega_{k}, k=$ $1,2, \ldots, K \leq[N / 2]$. The least squares estimate of the slope yields the differencing parameter $d=\hat{d}$ and $H=\hat{d}+0.5$. Next is how to set the value of $K$. Since, the differencing parameter $d$ is sensitive to the choice of $K$, we decided to keep it smaller than the standard value of $N^{0.5}$. We use $K=\left[N^{0.45}\right]=28$ in our analysis to determine the Hurst exponent, keeping in mind smaller powers of $N$ introduce large estimation errors. Once the differencing parameter $d$ was obtained, the Hurst exponent was obtained as $H=d+0.5$.

\section{APPENDIX B: RESULTS FROM HEMISPHERIC SUNSPOT NUMBERS}

The sunspot number is probably the longest data available to study solar activity. However, the hemispheric data of sunspot number were available only from 1992. Recently, hemispheric sunspot number data has been derived and made publicly available (Veronig et al. 2021) ${ }^{2}$ during 1874-2020. As the sunspot number has been extensively used
Table B1. Summary of results obtained for Higuchi's dimension and Hurst exponents from hemispheric sunspot numbers.

\begin{tabular}{llcc}
\hline \hline \multirow{2}{*}{ Method } & \multirow{2}{*}{$\begin{array}{l}\text { Window } \\
\text { (months) }\end{array}$} & \multicolumn{2}{c}{ Time Series } \\
\cline { 3 - 4 } & $\mathrm{AS}$ & $\mathrm{AS}_{\text {Norm }}$ \\
\hline Higuchi & $2-55$ & 1.90 & 1.90 \\
\hline Hurst (R/S) & $11-110$ & 0.83 & 0.83 \\
& $263-650$ & 0.93 & 0.83 \\
\hline Hurst (DFA) & $11-110$ & 0.90 & 0.89 \\
& $263-650$ & 1.07 & 0.88 \\
\hline Hurst (PR) & $\mathrm{K}=28$ & 0.73 & 0.76 \\
\hline
\end{tabular}

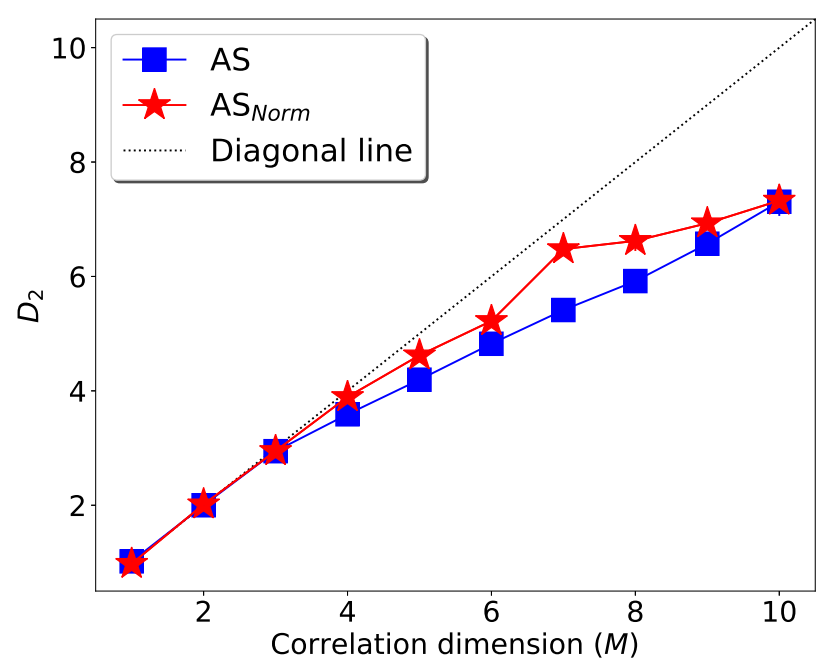

Figure B1. Variation of correlation dimension $\left(D_{2}\right)$ with the embedding dimension $(M)$ for $A S$ (square points) and $A S_{\text {Norm (asterisks) calculated }}$ from hemispheric sunspot numbers.

to study solar activity and it has a strong correlation with the sunspot area number, we present the results of our time-series analyses for the $A S$ and $A S_{N \text { orm }}$ computed from the sunspot number during the period May 1874 - September 2016. The results are presented in Table B1 and Figure B1.

This paper has been typeset from a $\mathrm{T}_{\mathrm{E}} \mathrm{X} / \mathrm{LAT} \mathrm{E} \mathrm{X}$ file prepared by the author.

\footnotetext{
2 https://wwwbis.sidc.be/silso/datafiles
} 